\title{
Supernova-Relevant Hydrodynamic Instability Experiment on the Nova Laser
}

J. Kane, D. Arnett, B. Remington, S. G. Glendinning, A. Rubenchik, J. Castor, and M. Berning

This paper was prepared for submittal to the Workshop in Astrophysics Experiments with Large Lasers Pleasanton, CA

February 26-27, 1996

February 12, 1996

This is a preprint of a paper intended for publication in a journal or proceedings. Since changes may be made before publication, this preprint is made available with the understanding that it will not be cited or reproduced without the permission of the author. 


\section{DISCLAIMER}

This document was prepared as an account of work sponsored by an agency of the United States Government. Neither the United States Government nor the University of California nor any of their employees, makes any warranty, express or implied, or assumes any legal liability or responsibility for the accuracy, completeness; or usefulness of any information, apparatus, product, or process disclosed, or represents that its use would not infringe privately owned rights. Reference herein to any specific commercial product, process, or service by trade name, trademark, manufacturer, or otherwise, does not necessarily constitute or imply its endorsement, recommendation, or favoring by the United States Government or the University of California. The views and opinions of authors expressed herein do not necessarily state or reflect those of the United States Government or the University of California, and shall not be used for advertising or product endorsement purposes. 


\section{DISCLAMMER}

Portions of this document may be illegible in electronic image products. Images are produced from the best available original document. 



\title{
Supernova-relevant hydrodynamic instability experiment on the Nova laser*
}

\author{
J. Kane, ${ }^{1}$ D. Arnett, ${ }^{1}$ B.A. Remington, ${ }^{2}$, S.G. Glendinning, ${ }^{2}$ \\ A.Rubenchik, ${ }^{3} \mathrm{~J}$. Castor, ${ }^{2}$ and M. Berning 4 \\ IUniv. of Arizona, ${ }^{2}$ Lawrence Livermore National Laboratory, \\ ${ }^{3}$ Univ. of Calif. Davis, ${ }^{4}$ Dusseldorf University \\ In preparation for presentation at the \\ Workshop on Astrophysics Experiments with Large Lasers \\ February 26-27, 1996, Pleasanton, CA, USA \\ and for submittal to the \\ Astrophysical Journal
}

\begin{abstract}
Supernova 1987A focused attention on the critical role of hydrodynamic instabilities in the evolution of supernovae. On quite a separate front, the detrimental effect of hydrodynamic instabilities in inertial confinement fusion (ICF) has long been known. Tools from both areas are being tested on a common project. At Lawrence Livermore National Laboratory (LLNL), the Nova Laser is being used in scaled laboratory experiments of hydrodynamic mixing under supernova-relevant conditions. Numerical simulations of the experiments are being done, using hydrodynamics codes at the Laboratory, and astrophysical codes successfully used to model the hydrodynamics of supernovae. A two-layer package composed of $\mathrm{Cu}$ and $\mathrm{CH}_{2}$ with a single mode sinusoidal 1D perturbation at the interface, shocked by indirect laser drive from the $\mathrm{Cu}$ side of the package, produced significant Rayleigh-Taylor (RT) growth in the nonlinear regime. The scale and gross structure of the growth was successfully modeled, by mapping an early-time simulation done with 1D HYADES, a radiation transport code, into 2D CALE, a LLNL
\end{abstract}


hydrodynamics code. The HYADES result was also mapped in $2 \mathrm{D}$ into the supernova code PROMETHEUS, which was also able to reproduce the scale and gross structure of the growth.

\section{INTRODUCTION}

A recent development in the field of astrophysics is the recognition of the occurrence of strong material mixing in Type II supernova (SN), driven by the Rayleigh-Taylor (RT) instability. The RT instability occurs at all stages of SN evolution. Buoyancy-driven convection in the first $100 \mathrm{~ms}$ provides the necessary heat transport to restart the stalled shock, allowing models of SN to explode.(Colgate, Herant, Benz article) In the first 6-12 months, RT spikes of heavy core material penetrate through the outer layers of the star,(Muller, Fryxell, Arnett) as illustrated by the image in Fig.la. This mixing of radioactive $\mathrm{Ni}$ and Co outwards provides a heat source to the outer regions of the star, thereby affecting the temporal evolution of the light curve. Much later in the time, as the SN proceeds into the remnant phase, the RT instability once again affects the evolution, causing material clumping often referred to as "bullets".(James Stone Nature article) Given the fundamental role played by the RT instability in SN evolution, it is desirable to develop the means of verifying the turbulent compressible mix modeling of the SN codes. To that end, we are developing an experiment using the Nova laser at Lawrence Livermore National Laboratory to study compressible RT mixing at relevant pressures. The tools for carrying out clean RT experiments had already been developed as part of the inertial confinement fusion effort at the Nova laser, as illustrated in Fig. 1b. The goal ultimately is to benchmark the fully $3 \mathrm{D}$ multimode RT mix modeling as applied in the SN codes. We describe a first step towards that goal here, 
where we use the SN code PROMETHEUS to model the 2D single-mode RT evolution into the deep nonlinear regime. Initial comparisons with the experiment look very favorable.

\section{EXPERIMENTAL CONFIGURATION}

The experimental configuration is illustrated in Fig. 2 and is described extensively elsewhere.(Remington et al., Phys. Plasmas, 1995). Eight of the ten Nova laser beams at a wavelength of $\lambda \quad L=0.351 \mu \mathrm{m}$, energy of 1.5 $\mathrm{kJ} / \mathrm{beam}$, and duration of $1 \mathrm{~ns}$ were focused into a $3.0 \mathrm{~mm}$ long, $1.6 \mathrm{~mm}$ diameter Au cylindrical radiation cavity, converting to a $\sim$ thermal $x$-ray drive. The experimental package is planar, a $80 \mu \quad \mathrm{m}$ thick $\mathrm{Cu}$ foil backed by $500 \mu \mathrm{m}$ of $\mathrm{CH}_{2} . \mathrm{A} \lambda=200 \mu \mathrm{m}$ wavelength, $\eta \quad{ }_{0}=20 \mu \mathrm{m}$ amplitude sinusoidal ripple is imposed at this imbedded interface. This is mounted across a 750 $\mu \mathrm{m}$ diameter hole in the hohlraum wall, so that the inner, smooth side of the $\mathrm{Cu}$ sees the $\mathrm{x}$-ray drive. Diagnosis of the interface is through side-on radiography. Two Nova beams at $\lambda \quad \mathrm{L}=0.528 \mu \mathrm{m}$, energy of $3 \mathrm{~kJ}$, and duration of $5 \mathrm{~ns}$ are focused onto a separate disk of $\mathrm{Fe}$, generating $\mathrm{He}-\alpha$ $\mathrm{x}$-rays at 6.7 $\mathrm{keV}$. The foil is then viewed edge-on as a shadow, with the $\mathrm{CH}_{2}$ being essentially transparent.

The laser pulse shape used in the experiments is illustrated in Fig. 3, and corresponds to a $1 \mathrm{~ns}$ square at total peak power of $12 \mathrm{TW}$. The resulting radiation temperature is also illustrated, and has a peak at about $210 \mathrm{eV}$ at $\sim 1 \mathrm{~ns}$, then monotonically falling off with time after the laser drive turns off. The radiation drive is characterized three different ways. On separate shots, a temporally resolved filtered photodiode array views a hole in the radiation cavity, thus directly measuring $\mathrm{x}$-ray flux.(Kornblum 
ref) A second technique is to mount a standard $\mathrm{Al}$ wedge ("witness plate") on the wall of the hohlraum, and measure the shock breakout times across various thicknesses of $\mathrm{Al}$, thus giving the shock trajectory.(Kauffman PRL). A resulting shock trajectory from this technique (for a 600 ps square laser pulse) is shown in Fig. 4. Finally, by simply viewing the position of the $\mathrm{Cu}$ $\mathrm{CH}_{2}$ interface as a function of time, one has a integral measure of the drive. All three techniques give similar results.

\section{1D SIMULATIONS}

The situation in the exploding star that we are attempting to simulate in the experiment is illustrated in Fig. 5a and b. In Fig. 5a we show the velocity of the He-H interface of an exploding Type II supernova. This 1D model corresponding to a star of initial mass of $25 \mathrm{M}^{*}$. After the Fe core collapse, a strong shock moves out through the star, providing the velocity impulse at $2500 \mathrm{sec}$. The He layer is then decelerated with time by the outer, lower density $\mathrm{H}$ layer. This decelerating interface is RT unstable, as illustrated in Fig. 5 b, since $\nabla \quad P \cdot \nabla \rho<0$, hence perturbations at the interface grow. The situation for the Nova laser experiment is illustrated in Fig. 5c and $d$. Though the spatial scales are vastly different, the pressures are comparable. Here the $\mathrm{Cu}-\mathrm{CH}_{2}$ interface is $\mathrm{RT}$ unstable in deceleration, similar to the He-H interface of the star.

In Fig. 6 we illustrate how we map the 1D radiation-hydrodynamics HYADES simulations at 2 ns into 1D CALE and 1D PROMETHEUS simulations. HYADES is a 1D Lagrangian code with multigroup radiation transport. We use the radiation temperature source shown in Fig. 3 as the input. Results from HYADES for density and pressure versus position at $20 \mathrm{~ns}$ are shown in 
Figs. $6 \mathrm{a}$ and $6 \mathrm{~b}$. The contact discontinuity between the $\mathrm{Cu}$ and $\mathrm{CH}_{2}$ is indicated by the arrow. At $2 \mathrm{~ns}$, all of the conditions from the HYADES calculation (pressure, density, temperature, velocity, etc.) are used as the starting conditions for the CALE and PROMETHEUS codes. The results at 20 ns from running these more sophisticated hydrodynamics codes from $2 \mathrm{~ns}$ on is shown in Fig. 6. Since the drive is rapidly turning off at $2 \mathrm{~ns}$, the hydrodynamics of what reaches the $\mathrm{Cu}$ interface $80 \mu \quad \mathrm{m}$ in from the ablation front is largely decoupled from the drive, and this mapping works very well. In other words, in isolating the RT unstable interface away from the ablation front, the instability evolution is governed only by compressible hydrodynamics.

\section{2D SIMULATIONS}

We use the same mapping scheme, but now as input to 2D CALE for a $\mathrm{Cu}-\mathrm{CH}_{2}$ interface that has a $\lambda \quad=200 \mu \mathrm{m}$ wavelength, $\eta \quad{ }_{0}=20 \mu \mathrm{m}$ amplitude sinusoidal ripple. The results of the 2D CALE simulation at 2, 5, 15, and 30 ns are shown in Fig. 7. At 2 ns, the time when the mapping from 1D HYADES takes place, we see that shock has not yet reached the thinnest point in the $\mathrm{Cu}$. By 5 ns the shock has just passed through the interface, and the ripple in the $\mathrm{Cu}$ is inverting phase, as expected by standard Richtmyer-Meshkov instability theory. By $15 \mathrm{~ns}$ the perturbation has grown with the opposite phase a modest amount, with the beginnings of a "mushroom head" forming at the tip of the spike. By $30 \mathrm{~ns}$, due to strong Kelvin-Helmholtz instability from the shear across the spike as it penetrates the $\mathrm{CH}_{2}$, a strong roll-up occurs on the RT spike. The overall peak-to-valley amplitude here is about $160 \mu \mathrm{m}$, which should be easily observable in side-on radiography in the experiment. 


\section{COMPARISON WITH EXPERIMENT}

In Fig. 8a we show 2D images from the full time sequence of the experiment. At $10 \mathrm{~ns}$, the perturbation has inverted phase, and RT spikes are just starting to form. At 15 and $20 \mathrm{~ns}$, the spikes continue to grow. By $25 \mathrm{~ns}$, the spikes have become considerably longer, with the spike tip obscured behind a resolution grid. At $30 \mathrm{~ns}$, on a laser shot with the resolution grid moved farther away from the interface, one can see indications of two roll-ups, one at the very tip of the interface, and one near its base.

In Fig. 8b, we show an enlarged version of the 30 ns image shown in 8a. In $8 \mathrm{c}$, we show the equivalent result from the $2 \mathrm{D}$ CALE simulation, and $8 \mathrm{~d}$ the same from PROMETHEUS. The gross features of the experiment are being well reproduced by both simulations, although neither simulation reproduces the second roll-up near the base of the RT spike.

In Fig. 9 we show the comparison of spike and bubble position from the experiment, CALE, and PROMETHEUS. Once again, the gross features of this 2D nonlinear RT instability experiment are being reproduced by both hydrodynamics codes. Future work will focus on making these comparisons more quantitative by including radiation transport and realistic EOS in PROMETHEUS. We will also investigate single-mode perturbations in 3D and multimode perturbations in 2D and 3D. We will ultimately look at a target with multiple layers, to investigate the effects of feedthrough and shock reverberations.

\section{REFERENCES}


*Work performed under the auspices of the U.S. Department of Energy by the Lawrence Livermore National Laboratory under contract number W7405-ENG-48.

\section{FIGURE CAPTIONS}

Figure 1: (a) 2D supernova simulation. (b) 3D Nova laser experiment.

Figure 2: Sketch of the experimental configuration.

Figure 3: Total laser power and deduced radiation temperature.

Figure 4: Measured and simulated shock position in $\mathrm{Al}$.

Figure 5: (a) 1D PROMETHEUS simulation showing the velocity of the $\mathrm{He}-\mathrm{H}$ interface. (b) PROMETHEUS result showing the density and pressure vs radius at $1000 \mathrm{sec}$. (c) HYADES simulation showing the velocity of the $\mathrm{Cu}$ $\mathrm{CH}_{2}$ interface in the laser experiment. (d) HYADES result for the density and pressure versus position in the laser exeriment.

Figure 6: 1D results from HYADES, CALE, and PROMETHEUS for (a) density vs position and (b) pressure vs position at $30 \mathrm{~ns}$, illustrating how the mapping from HYADES to CALE and PROMETHEUS is done at $2 \mathrm{~ns}$.

Figure 7: Results from a 2D CALE simulation for the evolution of a $\lambda \quad=200 \mu \mathrm{m}$ wavelength, $\eta_{\mathrm{o}}=20 \mu \quad \mathrm{m}$ amplitude sinusoidal ripple at the $\mathrm{Cu}-\mathrm{CH}_{2}$ interface. (a) $2 \mathrm{~ns}$, where the mapping from HYADES to CALE is done. (b) 5 ns showing the phase inversion. (c) $15 \mathrm{~ns}$, and (d) $30 \mathrm{~ns}$, showing prominent RayleighTaylor spike tip roll-up due to Kelvin-Helmholtz instability. 
Figure 8: (a) Full time sequence of the experiment, as viewed in side-on radiography. (b) Blow-up of the experimental image shown in (a) at $30 \mathrm{~ns}$. (c) Equivalent result at $30 \mathrm{~ns}$ from the 2D CALE simulation. (d) Equivalent result at $32 \mathrm{~ns}$ from the 2D PROMETHEUS simulation.

Figure 9: Spike and bubble position from the experiment vs time, compared with the results from the 2D CALE and PROMETHEUS simulations. 

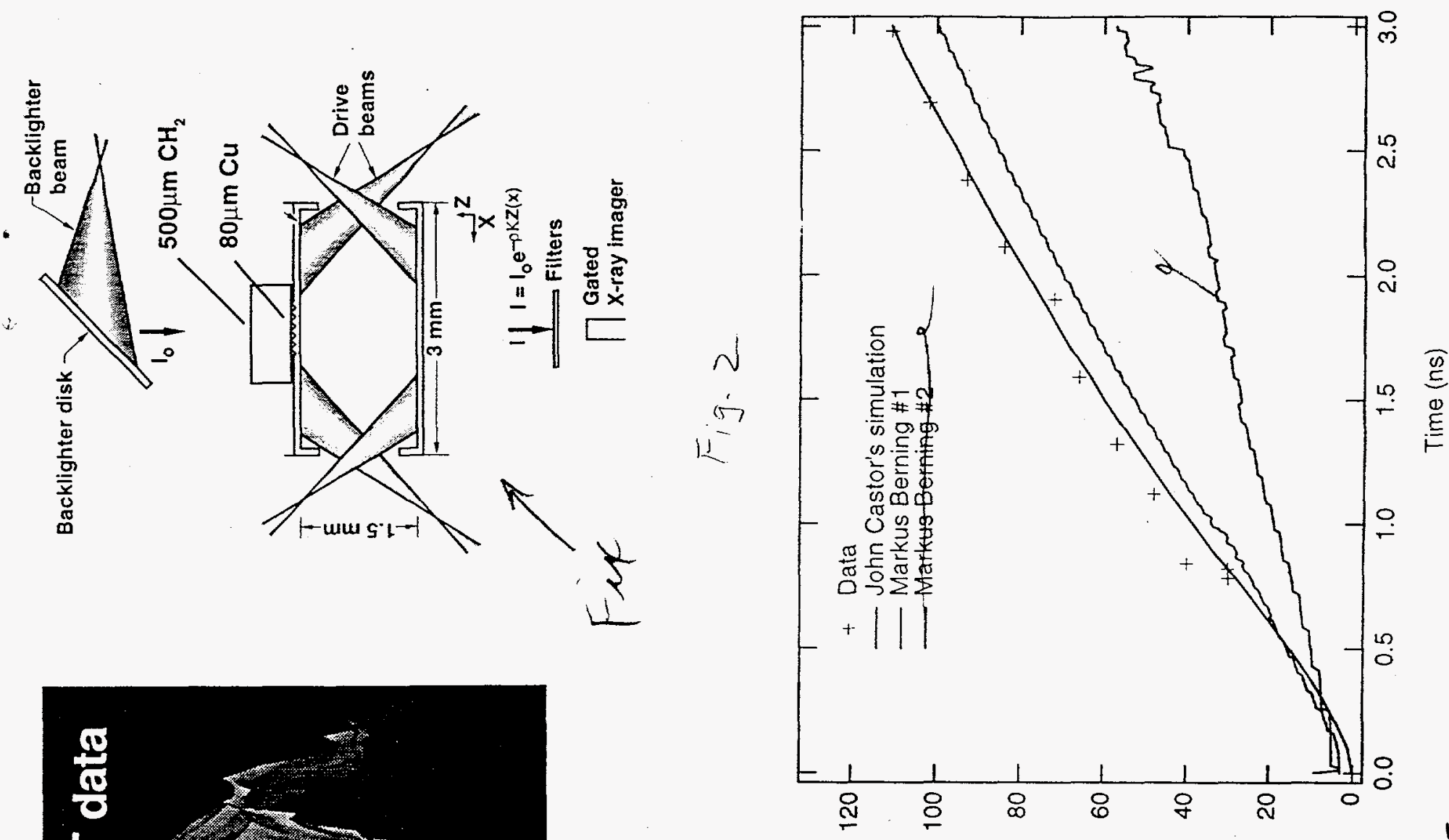

Q15

әбрәм u! uon!sod

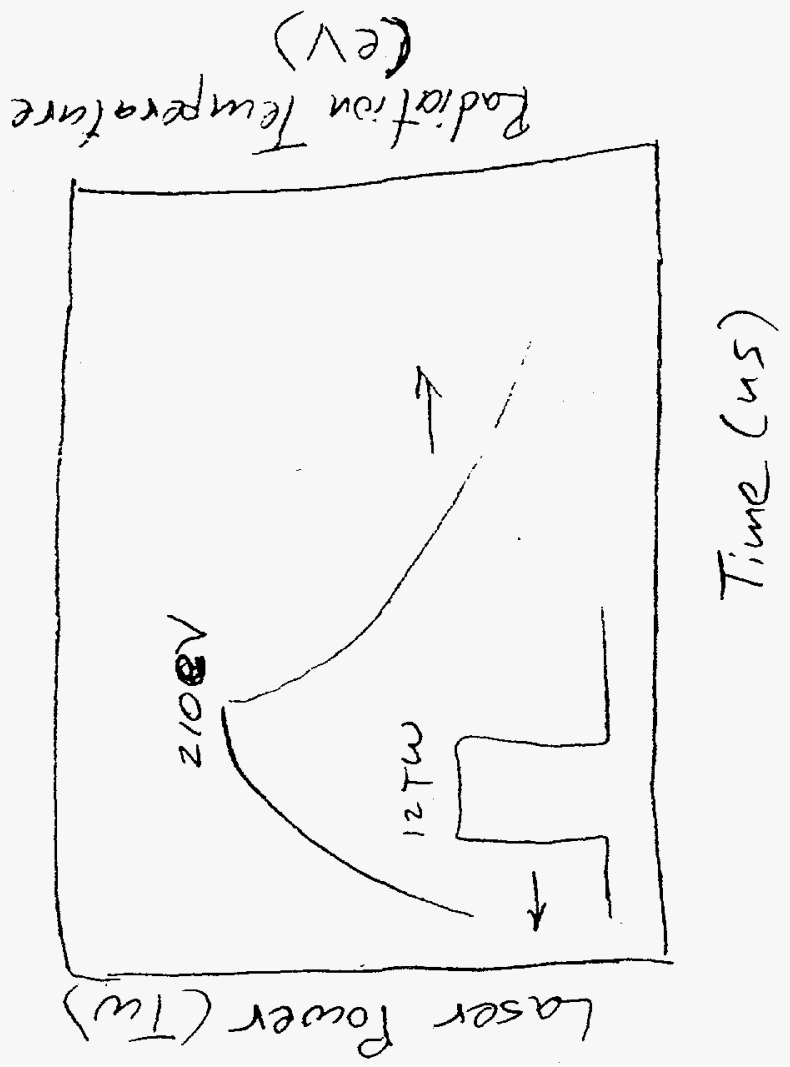


$\pi$

or
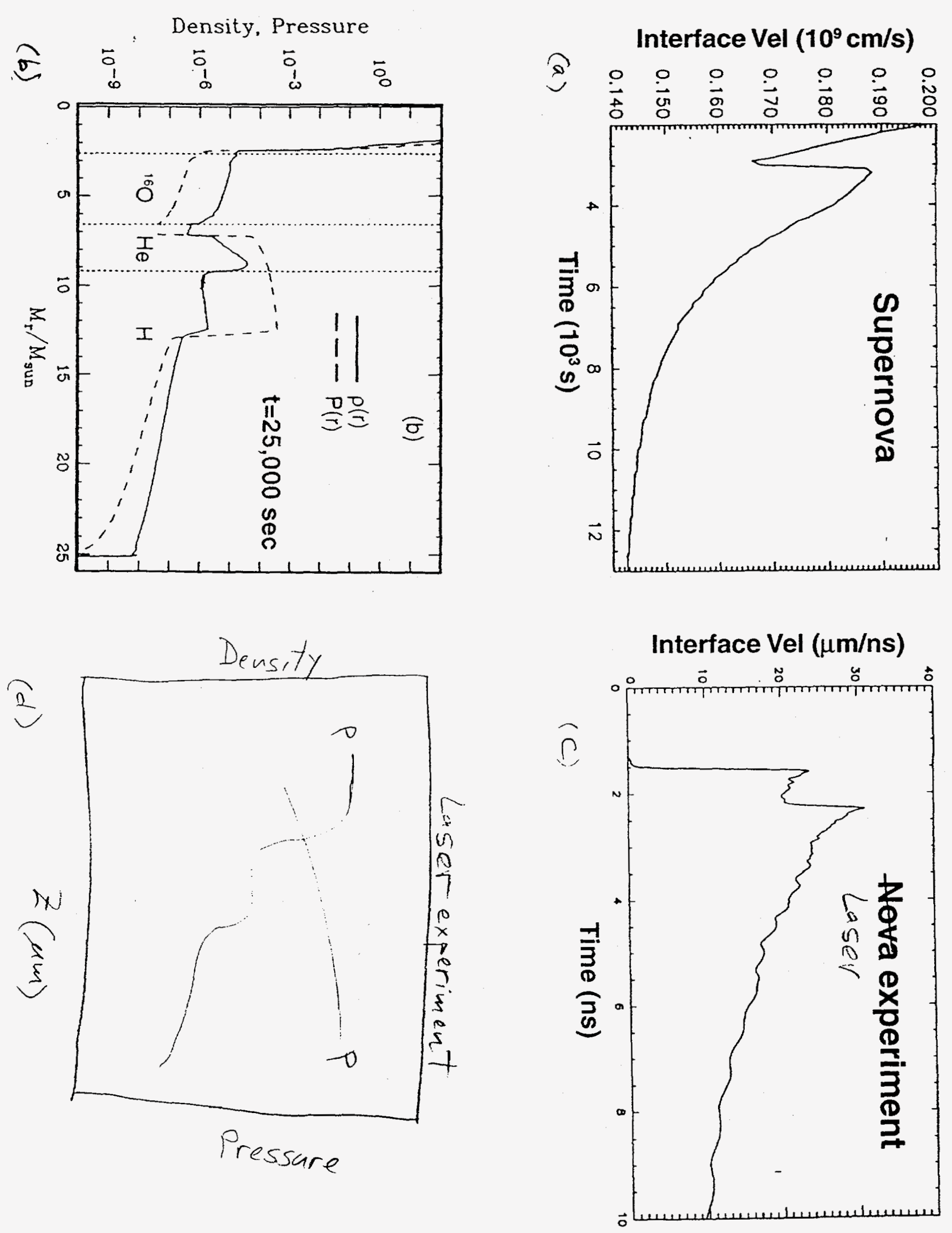

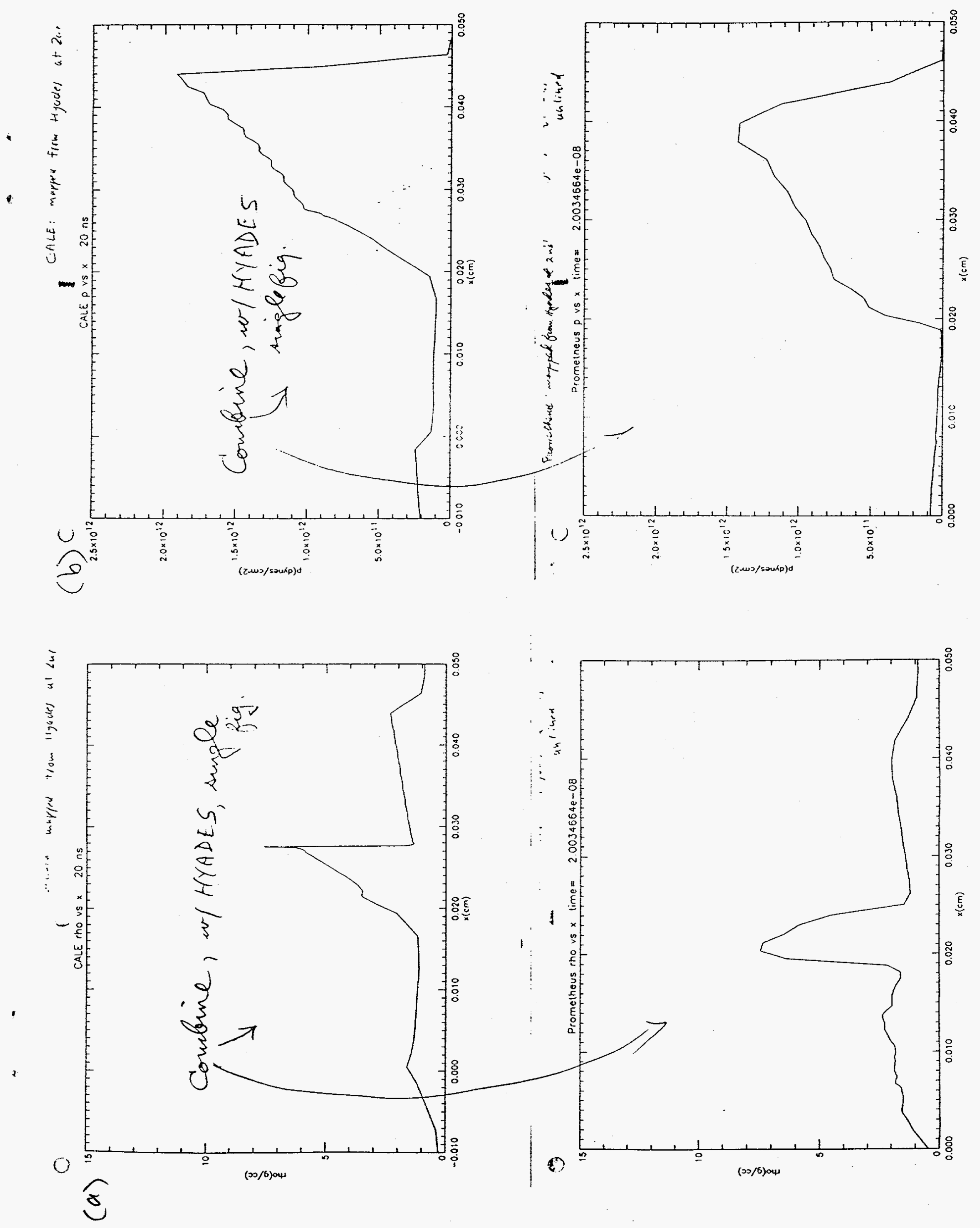

 
$\frac{T}{6}$

$\checkmark$
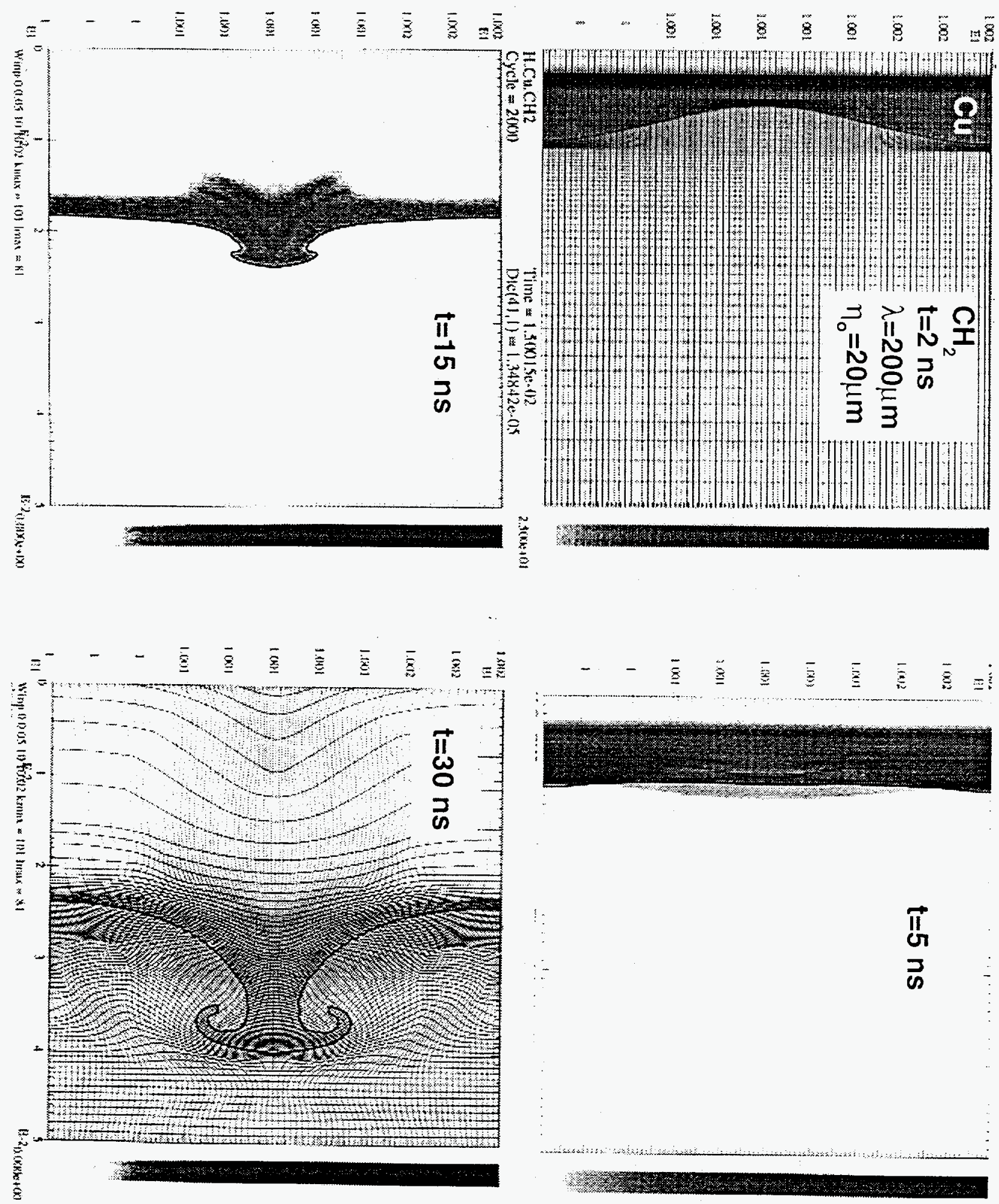

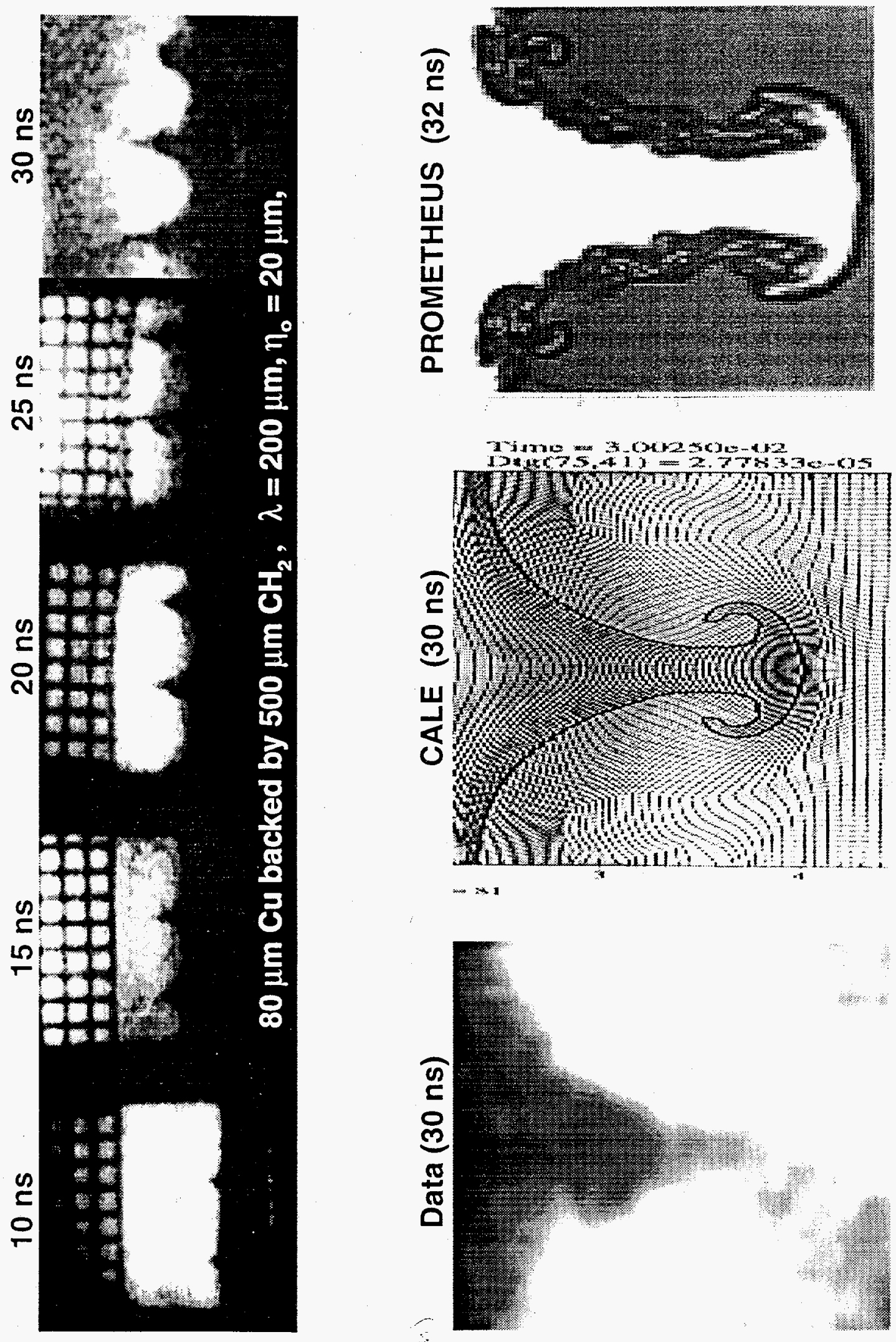


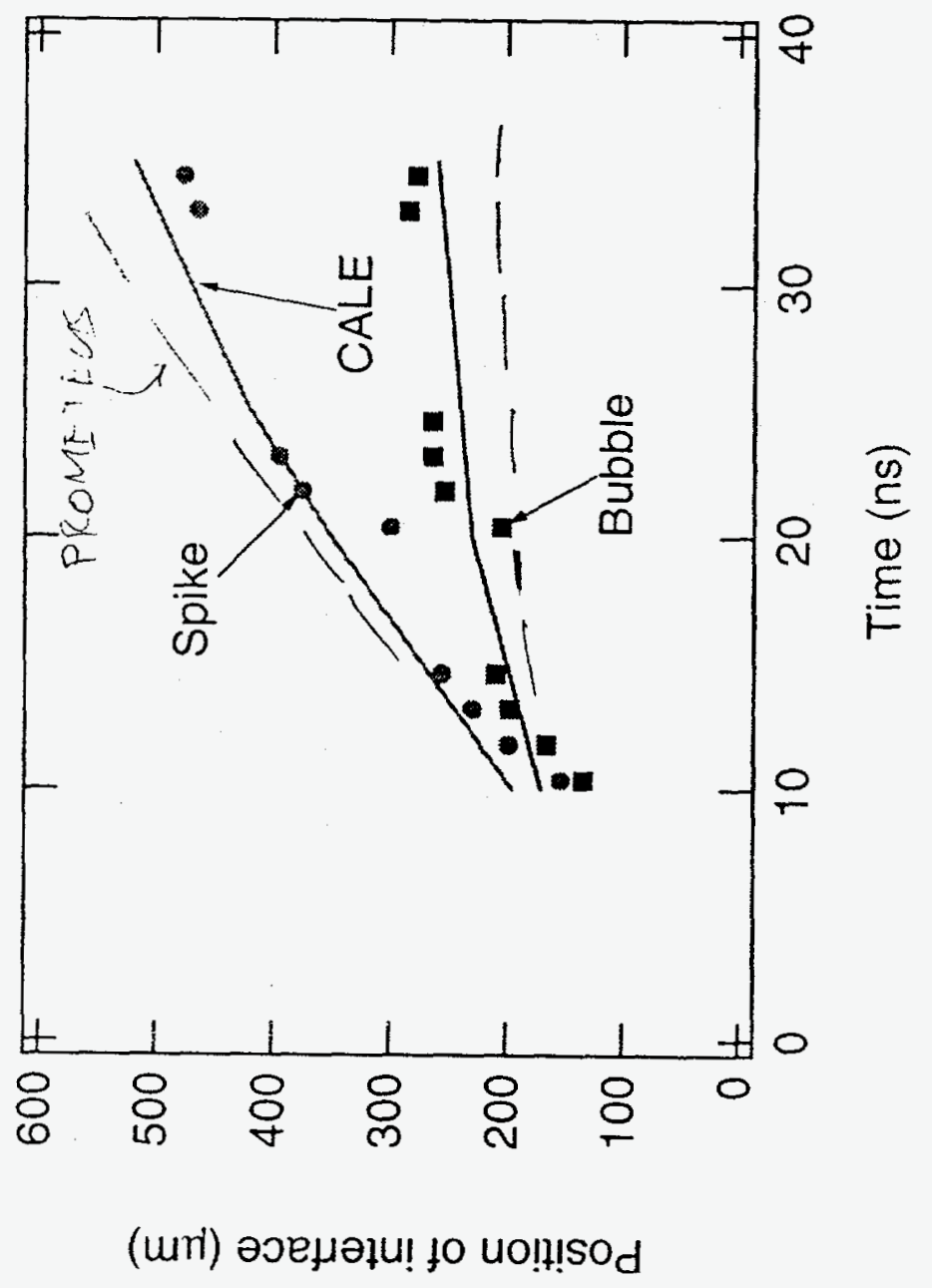

